eventually show signs of acute pancreatitis. ${ }^{3}$ On rare occasions, pancreatic tissues found in the hiatal hernia may undergo malignant transformation. ${ }^{4}$ Acute pancreatitis in these patients may be idiopathic or result from volvulus formation within the hernia. ${ }^{5}$ It is likely that multiple subclinical episodes of pancreatitis in the case described here led to adhesion of the pancreas to the posterior aspect of stomach and the adjoining areas of upper gastrointestinal tract. Thereafter, the pancreas ascended through the esophageal hiatus as a content of the hiatal hernia.

Complete herniation of the pancreas through the gastroesophageal hiatus is rare. Computed tomographic scan is effective for definitive diagnosis in this unusual condition. We believe that surgical repair of hiatal herniation of the pancreas is indicated even in symptom-free patients to prevent potential complications.

\section{References}

1. Loffeld RJ, van der Putten AB. Newly developing hiatus hernia: a survey in patients undergoing upper gastrointestinal endoscopy. J Gastroenterol Hepatol. 2002;17:542-4.

2. Katz M, Atar E, Herskovitz P. Asymptomatic diaphragmatic herniation of the pancreas. J Comput Assist Tomogr. 2002;26:524-5.

3. Gremmels JM, Broome DR, Fisher KL. Pancreatic herniation through the gastroesophageal hiatus: magnetic resonance imaging, magnetic resonance cholangiopancreatography, and computed tomography evaluation. J Comput Assist Tomogr. 2003;27:616-8.

4. Guillou L, Nordback P, Gerber C, Schneider RP. Ductal adenocarcinoma arising in a heterotopic pancreas situated in a hiatal hernia. Arch Pathol Lab Med. 1994;118:568-71.

5. Chevallier P, Peten E, Pellegrino C, Souci J, Motamedi JP, Padovani B. Hiatal hernia with pancreatic volvulus: a rare cause of acute pancreatitis. AJR Am J Roentgenol. 2001;177:373-4.

\title{
Duropleural fistula as a consequence of Gorham-Stout syndrome: A combination of 2 rare conditions
}

\author{
Ritwick Agrawal, MD, ${ }^{a}$ Imran Mohammed, MD, ${ }^{a}$ and Patrick G. Reilly, MD, \\ Pittsburgh, $\mathrm{Pa}$
}

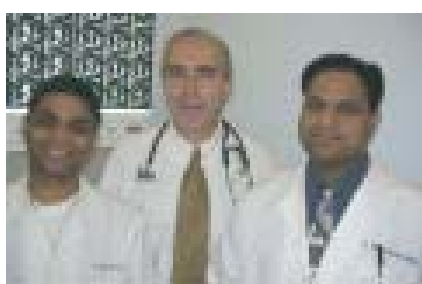

R. Agrawal, P.G. Reilly, I. Mohammed (left to right)

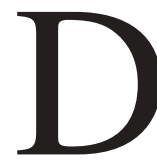
uropleural fistula (DPF) is an abnormal communication between the pleural and dural spaces caused by trauma, spinal surgery, or tumors. In our case it is caused by Gorham-Stout syndrome (GSS), a rare bone disease characterized by painless massive osteolysis caused by proliferation of lymphangiomatous tissue. The spinal form of GSS is associated with kyphosis, kyphoscoliosis, subluxation, and dislocation. DPF has never been previously described in association with GSS.

\section{Clinical Summary}

A 25-year-old woman with a history of Chiari I malformation was admitted with 2 days' duration of low-grade fever, fatigue, and dysphagia. Over the past 1 year, she had undergone multiple suboccipital craniotomies. Emergency magnetic resonance imaging of the brain did not show any change in size or dimension of the fourth ventricle. Physical examination was unremarkable ex-

From the Departments of Medicine ${ }^{\mathrm{a}}$ and Pulmonary and Critical Care, ${ }^{\mathrm{b}}$ Mercy Hospital of Pittsburgh, Pittsburgh, Pa.

Received for publication Dec 22, 2005; accepted for publication Jan 13, 2006.

Address for reprints: Imran Mohammed, MD, Department of Medicine, Mercy Hospital of Pittsburgh, 1400 Locust St, Pittsburgh PA 15219 (Email: doc_imran@yahoo.com).

J Thorac Cardiovasc Surg 2006;131:1205-6

$0022-5223 / \$ 32.00$

Copyright $\odot 2006$ by The American Association for Thoracic Surgery doi:10.1016/j.jtcvs.2006.01.023 cept for dullness to percussion and rhonchi in the left lower lung fields. Chest radiography revealed a left lower lobe infiltrate and moderate left-sided pleural effusion. She was treated empirically with intravenous piperacillin/tazobactam for aspiration pneumonia.

Two days later, she experienced respiratory failure, necessitating intubation and ventilator support. A computed tomographic scan of the chest showed destruction of the left posterior T7 vertebral body and left-sided ribs, 5th through 9th, which was suspicious of a malignant process. Pleural fluid analysis was bloody in appearance, with a lactate dehydrogenase level of 211 IU/L, a pleural/serum lactate dehydrogenase ratio of 0.88 , a pleu$\mathrm{ral} / \mathrm{serum}$ protein ratio of 0.57 , a white cell count of $5900 / \mu \mathrm{L}$ (mononuclear cells, 57\%; lymphocytes, 34\%), a glucose level of $103 \mathrm{mg} / \mathrm{dL}$, and an exudative pleural effusion. Thoracic myelography revealed an extravasation of contrast media, confirming a diagnosis of DPF centered at T7 and destructive lesions from the T6 through T8 vertebrae (Figure 1, $A$ ).

The patient underwent T6 through T8 laminectomy with duroplasty and DPF repair with a collagen matrix patch. Histopathologically, the T7 lytic lesion showed an irregular angiomatous proliferation in bone trabeculae and reactive bone formation, along with massive osteolysis, which is diagnostic of GSS (Figure 2). She was treated with intravenous vitamin D, followed by 50,000 IU of oral vitamin D weekly. Six-week follow-up thoracic myelography did not show any evidence of DPF (Figure 1,B).

\section{Discussion}

To our knowledge, we are reporting the first case of DPF caused by GSS. It is a consequence of blunt or penetrating trauma to the spine, complication of neurosurgical procedures, or tumors. ${ }^{1}$ Pres- 

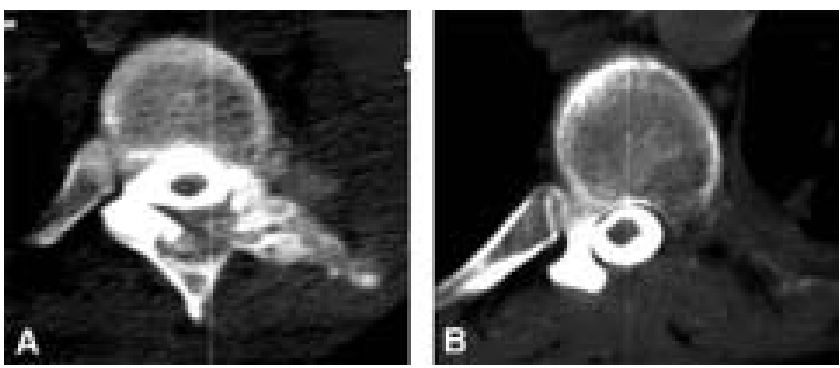

Figure 1. CT myelogram. A, Extravasation or dye suggesting duropleural fistula. B, Post-operative resolution of duro-pleural fistula.

sure gradients between the negative pleural pressure and positive dural pressure keeps the fistulous tract patent. ${ }^{2}$

DPF usually presents with symptoms of pleural effusion. The severity of disease depends on the duration and acuity of the fistula formation. It might present as an asymptomatic small pleural effusion or massive pleural effusion causing respiratory distress. Our case was diagnosed because of a high index of suspicion. The worsening respiratory failure and lack of response to antibiotics provided some additional clues. Lytic lesions in the bone and the location of the fistula, which was distant from the regions of previous operations, ruled out iatrogenic fistulous complications. The pleural effusion in DPF is usually transudative; however, bleeding, infections, and malignancy can coexist and form an exudate. The exact cause of exudative pleural effusion in our case is unknown; however, possible mechanisms include parapneumonic process, rupture of microvascular channels, or use of diuretics.

A presumptive diagnosis can be made on the basis of the presence of $\beta_{2}$ transferrin $^{3}$ in the pleural fluid. It is produced exclusively in the brain and inner ear perilymph, although this finding might be false negative ${ }^{2}$ in some cases. Definitive diagnosis can be made by means of myelography or radionucleotide myelography.

The management of DPF depends on the specific case. It can be treated conservatively with thoracentesis or surgically by repairing the fistula and duroplasty. Pollack and colleagues ${ }^{4}$ reviewed 19 cases of traumatic DPF: 13 responded to laminectomy and thoracotomy with duroplasty, and 6 were treated conservatively with chest tube drainage or thoracentesis. The exact timing of surgical intervention is debated, with variable opinions of early intervention versus a trial of chest tube drainage followed by surgical intervention.

GSS is a rare disease, with less than 150 cases reported thus far. ${ }^{5}$ Pathologically, it involves abnormal proliferation of capillaries

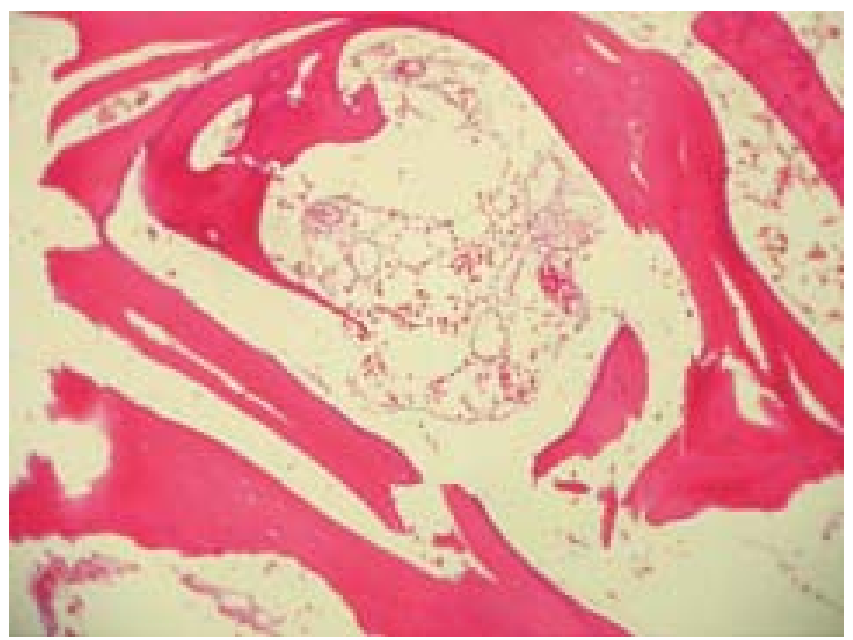

Figure 2. Biopsy of bone demonstrating proliferation of thinwalled vascular channels and osteolysis (hematoxylin-eosin, original magnifications, $100 \times$ ).

or sinusoidal channels of vascular or lymphatic origin and massive osteolysis. Only 29 cases of spinal GSS have been reported, ${ }^{5}$ largely causing deformities like kyphosis, kyphoscoliosis, subluxation, and dislocation. There are 15 cases of chylothorax, and 9 cases of pleural effusion that have been reported with GSS. ${ }^{5}$ GSS has a biologic heterogeneous pattern and includes a spectrum of lesions that can either regress spontaneously, remain unchanged for years, or develop into a recalcitrant form. Treatment modalities include radiation, vitamin D supplementation, antiosteoclastic medication, and $\alpha-2 b$ interferon. Surgical options are resection of the lesion, with reconstruction with a bone graft, a prosthesis, or both.

\section{References}

1. Qureshi MM, Roble DC, Gindin RA, Scudamore HH. Subarachnoidpleural fistula. Case report and review of the literature. $J$ Thorac Cardiovasc Surg. 1986;91:238-41.

2. Lloyd C, Sahn SA. Subarachnoid pleural fistula due to penetrating trauma: case report and review of the literature. Chest. 2002;122: 2252-6.

3. Huggins JT, Sahn SA. Duro-pleural fistula diagnosed by beta2-transferrin Respiration. 2003;70:423-5.

4. Pollack II, Pang D, Hall WA. Subarachnoid-pleural and subarachnoidmediastinal fistulae. Neurosurgery. 1990;26:519-25.

5. Patel DV. Gorham's disease or massive osteolysis. Clin Med Res. 2005;3:65-74. 